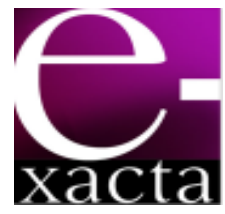

ISSN: 1984-3151

\title{
AutomaÇÃo Predial COM UTILIZAÇÃo de CONTROLADOR LÓGICO PROGRAMÁVEL
}

\section{Building Automation using Programmable Logic Control}

\author{
Breno Monteiro ${ }^{1 *}$; Bruno Viana ${ }^{1}$; Guilherme Camelo'; \\ Jurandir Agostinho' ${ }^{1}$; Lilian de Melo'; Marcelo Faleiro'; \\ Rafael Zanini ${ }^{1}$; Rodrigo Barbosa ${ }^{1}$; Euzébio D. Souza ${ }^{2}$ (Orientador)
}

1 Alunos de Graduação em Engenharia Elétrica do Centro Universitário de Belo Horizonte - UniBH. Belo Horizonte, MG. "breno.souza.monteiro@vale.com.

2 Especialista em Engenharia de Segurança do Trabalho FEAMIG, 1996. Professor do Centro Universitário de Belo Horizonte - UniBH. Belo Horizonte, MG. euzebio.souza@prof.unibh.br.

Recebido em: 19/08/2011 - Aprovado em: 11/10/2011 - Disponibilizado em: 13/10/2011

RESUMO: Para o controle dos circuitos de iluminação e circuitos de temperatura predial, é muito comum, encontrar em vários estabelecimentos brasileiros, o uso de diversos sistemas independentes de automação com a utilização de sensores que são totalmente controlados por seus proprietários, que além de gerar transtornos e esquecimentos quanto a seus acionamentos, podem com isto acarretar possíveis desperdícios de energia elétrica. Alguns aspectos importantes como temperatura, cor das lâmpadas, rendimento das luminárias, rendimento do reator utilizado e eficiência luminosa das lâmpadas são de extrema importância para um projeto que visa obter resultados voltados para a eficiência energética. Este artigo tem como objetivo, propor a automatização do acionamento dos circuitos de iluminação e ventiladores das salas de aula do Centro Universitário de Belo Horizonte - UniBH, visando a eficientização energética em termos de: redução do consumo de energia, custo de manutenção, maior vida útil das lâmpadas e maior eficiência luminosa. Pretende-se propor com a adoção do CLP uma redução significativa no consumo de energia, apenas evitando o desperdício.

PALAVRAS-ChaVe: CLP. Desperdício. Automação. Consumo. Custo.

ABSTRACT: For the control of lighting circuits and circuits building temperature, it is very common, found in various Brazilian institutions, the use of several independent systems of automation with the use of sensors that are fully controlled by their owners, who in addition to generating and disorders forgetting about your drives, it can lead to possible waste of energy. Some important aspects such as temperature, color lamps, luminaire efficiency, performance and efficiency of the reactor of the light bulbs are extremely important for a project that aims to achieve results toward energy efficiency. This article aims to propose the automation of the drive circuits of lighting and fans of the classrooms of the Centro Universitário de Belo Horizonte - UniBH, aimed at energy efficiency in terms of: reduction of energy consumption, maintenance cost, longer life of light bulbs and more efficient. It is intended to propose the adoption of the CLP a significant reduction in energy consumption, avoiding waste only. KEYWORDS: PLC. Waste. Automation. Consumption. Cost.

\section{INTRODUÇÃO}

Com a proposta de melhorar a qualidade de vida, aumentar a segurança, o bem estar, a redução nos afazeres domésticos, bem como diminuição de custos, surgiu a domótica, termo que, é uma fusão da palavra 
latina domus (casa) e do moderno, robótica. A domótica é um novo domínio de aplicação tecnológica, visa uma utilização racional e planejada dos diversos meios de consumo.

A domótica tem interessado aos clientes pessoas físicas, assim deixando de ser uma área exclusivamente das empresas do ramo da construção, cada vez mais oferecendo soluções, proporcionando maior conforto, segurança e também a redução nos gastos de energia elétrica e água potável.

A proposta deste trabalho é fugir dos sistemas domóticos clássicos com alto valor de desenvolvimento e instalação, projetando um sistema de automação predial de baixo custo. Baseado em conceitos difundidos nas indústrias, com a utilização de um CLP, sensores e atuadores discretos, de fácil aquisição junto ao mercado, que atuarão na supervisão, controle e monitoramento de equipamentos como ventiladores e sistemas de iluminação, este projeto visa automatizar a iluminação e climatização de uma sala de aula conforme parâmetros externos, luminosidade e temperatura ambiente.

\section{Desenvolvimento}

Elaborar um estudo voltado para a ergonomia e conforto visual, para reduzir o consumo de energia de certo estabelecimento não é uma tarefa muito simples. Neste contexto, o presente artigo busca apresentar um estudo de automatização predial das salas de aula do Centro Universitário de Belo Horizonte - Uni-BH, focando na atuação dos circuitos elétricos conforme a exigência do ambiente, de modo a propor melhorias para a organização.

Foram montados três circuitos de iluminação independentes e um circuito de refrigeração.

Os circuitos de iluminação consistem em três modos que serão apresentados abaixo:
- Circuito 01 representará uma situação onde ocorra a influência de alta iluminação externa fazendo com que apenas $1 / 3$ das lâmpadas acendam;

- Circuito 02 representará uma situação onde ocorra a influência de média iluminação externa fazendo com que $2 / 3$ das lâmpadas acendam;

- Circuito 03 representará uma situação onde ocorra a influência de baixa iluminação externa fazendo com que todas as lâmpadas se acendam;

O circuito de refrigeração funcionará da seguinte maneira: se o ar-condicionado estiver ligado e a porta da sala de aula ficar aberta por um tempo determinado de 3 minutos então automaticamente 0 arcondicionado desligará, porém se a porta for fechada antes do tempo estimado, o mesmo continuará em funcionamento normal. Se o ar- condicionado for desligado pela contagem de tempo estimado pela lógica do programa, o mesmo entrará em funcionamento somente quando a porta da sala de aula estiver fechada e após uma nova contagem de tempo.

Lembrando que estas situações funcionarão se o CLP estiver em modo automático, pois se o mesmo estiver em modo manual os circuitos funcionarão como o usuário preferir.

Para alcançar este objetivo é necessário realizar o projeto de automação. Primeiro precisamos identificar o sistema que se deseja automatizar ou controlar e modelar matematicamente este sistema. Em segundo lugar, definir o controlador deste sistema, definindo as ações de controle, os sensores e os atuadores.

\section{Conceitos}

Segundo (NATALE, 2003) o Controlador Lógico Programável é um equipamento eletrônico digital com 
hardware e software compatíveis com aplicações industriais e residenciais.

Conforme (ANTONELLI, 2011), a norma NEMA interpreta o CLP, como sendo um aparelho eletrônico digital que utiliza uma memória programável para armazenar internamente instruções e programar funções específicas, tais como lógica, sequenciamento, temporização, contagem e aritmética, controlando, por meio de módulos de entradas e saídas, vários tipos de máquinas ou processos.

\section{Vantagens e desvantagens do uso de CLPs}

As vantagens associadas ao uso do CLP quando comparado com os painéis eletromecânicos são as seguintes:

- ocupam menor espaço;

- requerem menor potência elétrica;

- $\quad$ podem ser reutilizados;

- são programáveis, permitindo alterar os parâmetros de controle;

- apresentam maior confiabilidade;

- manutenção mais fácil e rápida;

- oferecem maior flexibilidade;

- permitem maior rapidez na elaboração do projeto do sistema.

As desvantagens no uso do CLP são as mesmas associadas aos sistemas domóticos centralizados de controle e podemos citar:

- grande quantidade de cabeamento;

- centralização das funções;

- complicada interface homem - máquina.

Embora o sistema eletromecânico em pequenas e até médias aplicações possa apresentar um custo inicial menor, esta vantagem poderá ser perdida considerando-se a relação custo/benefício que o CLP proporciona (SOUZA, 2001).

\section{Sensores e atuadores}

Conforme (NATALE, 2003)

"sensores são dispositivos que mudam seu
comportamento sob a ação de uma grandeza
física, podendo fornecer diretamente ou
indiretamente um sinal que representa uma
proporção da variação desta grandeza".

Os sensores comumente utilizados para automação de sistemas são:

- fim de curso;

- sensor de luminosidade;

- botoeiras;

- sensor óptico;

- sensor de proximidade;

- caracteristicas do CLP

- descrição de Funcionamento da automatização proposta

- o software foi desenvolvido utilizando o programa da Metaltex, IG Soft. Neste software é possível o desenvolvimento de todo 0 programa, podendo imprimir o programa de comutação, armazená-lo em arquivo específico, estando sempre ao alcance do desenvolvedor para possíveis modificações. Sendo que durante a edição do programa todas as linhas de conexão e dos blocos de contato estarão na cor preta.

- utilizou-se o CLP da Metaltex GPM-18R com interface homem-máquina (IHM) incorporada possui $18 \mathrm{E} / \mathrm{S}$ sendo 10 entradas digitais e 8 saídas digitais a relé ou transistor. Entre as entradas há 2 entradas de contagem rápida $200 \mathrm{KHz}$ e entre as saídas do modelo a transistor 2 saídas rápidas de até $400 \mathrm{KHz}$. 
Porta de programação RS232 e porta de comunicação RS485 que também comunica em Modbus RTU mestre ou escravo.

- um módulo de expansão pode ser adicionado com entradas analógicas para PT100, corrente ou tensão, bem como saída analógica. Outra opção de expansão seria através de remotas Modbus (MA), até 16 módulos com E/S analógicas ou digitais. A IHM é de LCD gráfica e suas teclas são sensíveis a toque, inclusive além das teclas predefinidas podem ser configuradas livremente teclas no display. A programação do CLP é feita através do GP - Software (programação em ladder) e a IHM através do IG-Software, ambos em português.

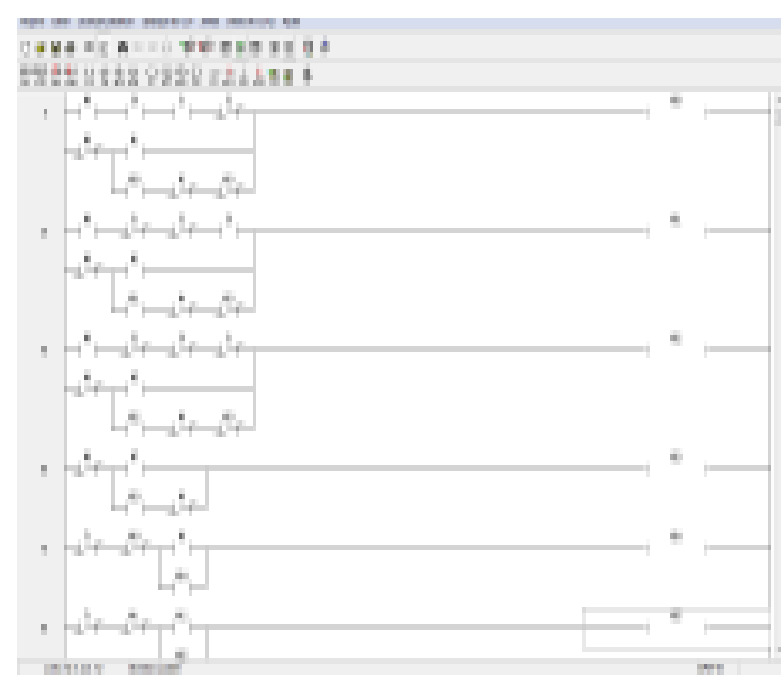

Figura 1 - Tela de edição do CLP. Fonte - Software Metaltex, IG Soft.

Segue a relação das entradas e saídas utilizadas para o desenvolvimento do projeto.

\section{Tabela 1}

Código das entradas do CLP e designações.

\begin{tabular}{c|l}
\hline $\begin{array}{c}\text { Código das } \\
\text { Entradas }\end{array}$ & \multicolumn{1}{|c}{ Designação do Programa } \\
\hline X0 & Sinal de baixa de luminosidade \\
X1 & Sinal media luminosidade \\
X2 & Sinal de alta luminosidade \\
X3 & Suíte NBR 5413 da porta \\
\hline
\end{tabular}

Fonte - Projeto Elétrico Detalhado.

Tabela 2

Código das saídas do CLP e designações

\begin{tabular}{c|c}
\hline $\begin{array}{c}\text { Código das } \\
\text { Saídas }\end{array}$ & Designação do Programa \\
\hline Y0 & Circuito para baixa luminosidade \\
Y1 & Circuito para média luminosidade \\
Y2 & Circuito para alta luminosidade \\
Y3 & Circuito modo apresentação \\
Y4 & Circuito ventilador \\
\hline
\end{tabular}

Fonte - Projeto Elétrico Detalhado.

\section{LógICA DE CONTROLE}

\section{Linguagem Ladder}

A linguagem de programação LADDER possui todos os elementos necessários para a criação de um programa completo. A programação LADDER permite ao técnico estruturar a sua programação claramente através de caixas de Funções e Blocos de Funções. Estruturar um programa consiste em dividir um programa de estrutura mais complexa em pequenos programas de estrutura mais simples. As estruturas simples são programadas em objetos criados pelo programador, tipo Blocos de Funções ou caixas Funções.

Na FIG 2 e na FIG 3 são apresentados os diagramas em blocos representando a lógica utilizada. 


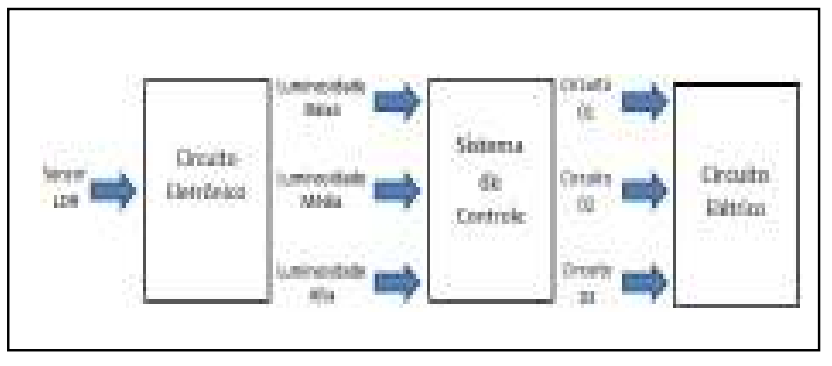

Figura 2 - Diagrama em blocos do sistema.

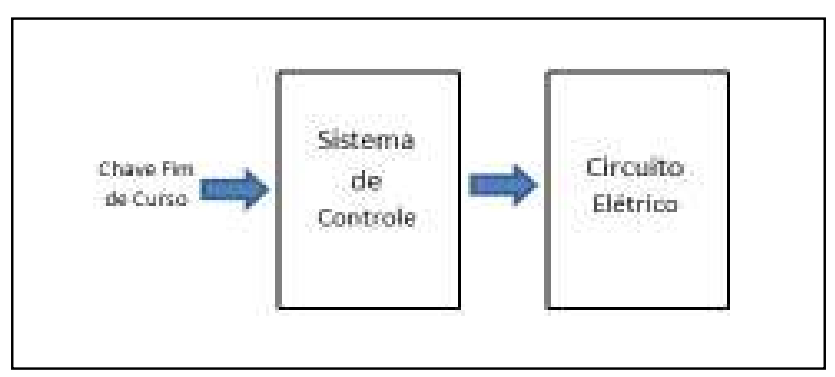

Figura 3 - Diagrama em blocos do sistema.

\section{INTERFACES GRÁFICAS}

Interface Gráfica (IHM) é um conceito da forma de interação entre o usuário do computador e um programa por meio de uma tela ou representação gráfica, visual, com desenhos, imagens, etc. Geralmente é entendido como a "tela" de um programa. Nas FIG 4 a FIG 8 seguem a ilustração das telas usadas pelo programa do CLP para comandar o sistema de iluminação e refrigeração.

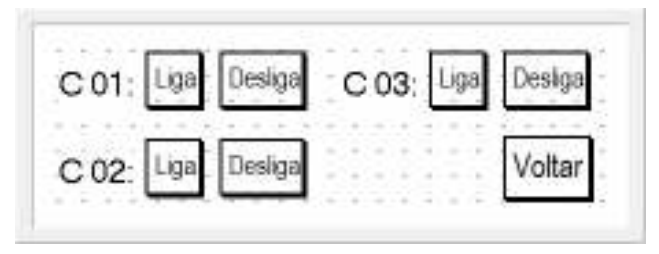

Figura 4 - Interface dos Circuitos

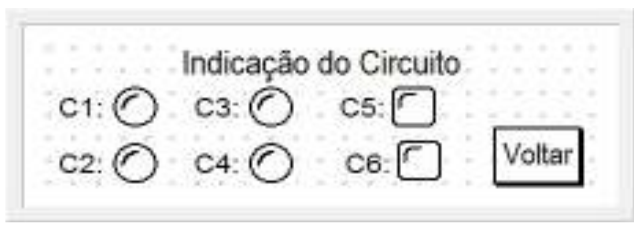

Figura 5 - Interface de Indicação dos Circuitos

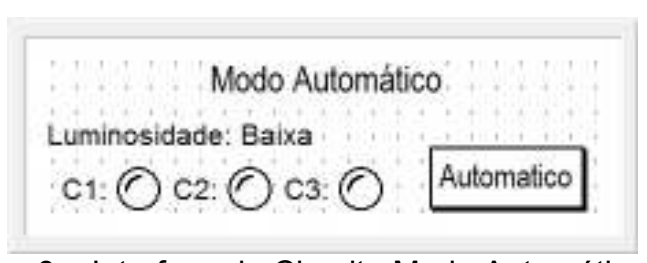

Figura 6 - Interface do Circuito Modo Automático

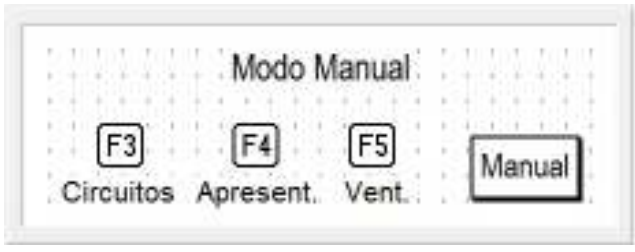

Figura 7 - Interface do Circuito Modo Manual

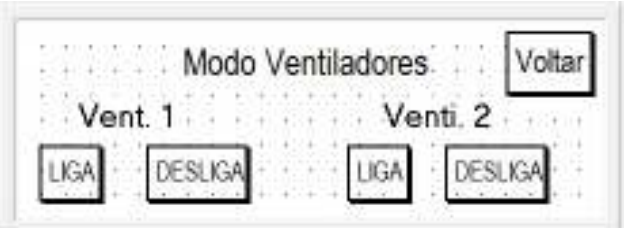

Figura 8 - Interface do Circuito Modo Ventiladores

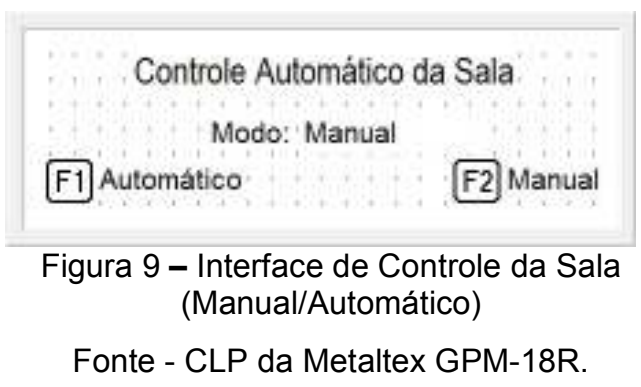

\section{CuSTOS DE IMPLANTAÇÃo}

A princípio, o investimento inicial deste novo sistema é elevado, mas a economia e conforto serão satisfatórios. Não podemos calcular o tempo para retorno do investimento, pois o funcionamento dos circuitos dependerá do usuário para mantê-lo em automático e também das condições externas, ou seja, da iluminação externa ou comando automático do ar-condicionado.

Mais do que seguir tendência, a automação reduz de forma significativa as despesas com energia elétrica, já que é possível utilizá-la de forma mais racional. 
Sem contar que o usuário ganha em qualidade de vida, pois muitas preocupações podem ser evitadas se programadas com antecedência. Em meio à "correria" é muito bom contar com a ajuda dos recursos tecnológicos e ainda poder ter um imóvel mais bonito e valorizado.

Os preços na TAB. 3, aqui apresentados foram pesquisados em lojas de materiais elétricos e eletrônicos da região, além de Internet, para que os preços médios fossem os mais próximos da realidade. O orçamento aqui apresentado não está considerando cabos, tubulações e mão de obra.

Tabela 3

Custo dos componentes

\begin{tabular}{c|c|c}
\hline Equipamento & Descrição & Preço Unitário \\
\hline CLP & $\begin{array}{c}\text { CLP GPM-18r } \\
\text { METALTEX } \\
\text { Limit Switches } \\
\text { Chave de Fim de } \\
\text { Curso } \\
\begin{array}{c}\text { Sensor De } \\
\text { Luminosidade }\end{array}\end{array}$ & $\mathrm{R} \$ 1098,00$ \\
\hline \multicolumn{2}{c|}{ Valor Total: } & $\mathrm{R} \$ 15,00$ \\
\hline \multicolumn{2}{c|}{} & $\mathrm{R} \$ 1123,00$
\end{tabular}

Fonte - Projeto Elétrico Detalhado.

\section{6 - EXPERIMENTO}

A partir do conceito detalhado anteriormente, foi elaborado um protótipo representando a sala de aula 206 do bloco A2 do Centro Universitário de Belo Horizonte - UniBH. Baseado nas condições reais de luminosidade e temperatura presentes no ambiente em estudo foi desenvolvido um circuito, como na FIG 10, em menor escala capaz de simular o funcionamento esperado

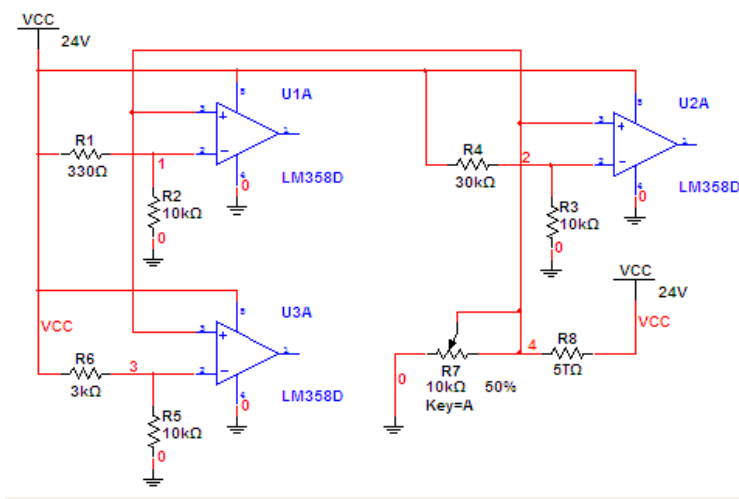

Figura 10 - Circuito do sensor de luminosidade.

Abaixo são listados os componentes utilizados na maquete, cujo esboço está na FIG 11 e FIG 12, ilustrando a condição esperada da realidade:

- 12 lâmpadas de $24 \mathrm{~V}$;

- 1 PLC METALTEX GPM-18R;

- 2 coolers, para representar o ar-condicionado;

- 1 Micro Switch (fim de curso);

- sensor de luminosidade (montado pelo grupo um circuito com um LDR utilizando amplificadores operacionais).

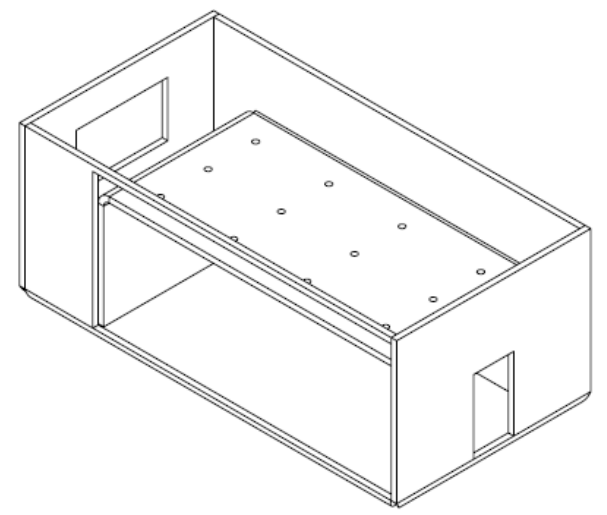

Figura 11 - Representação gráfica da maquete. 


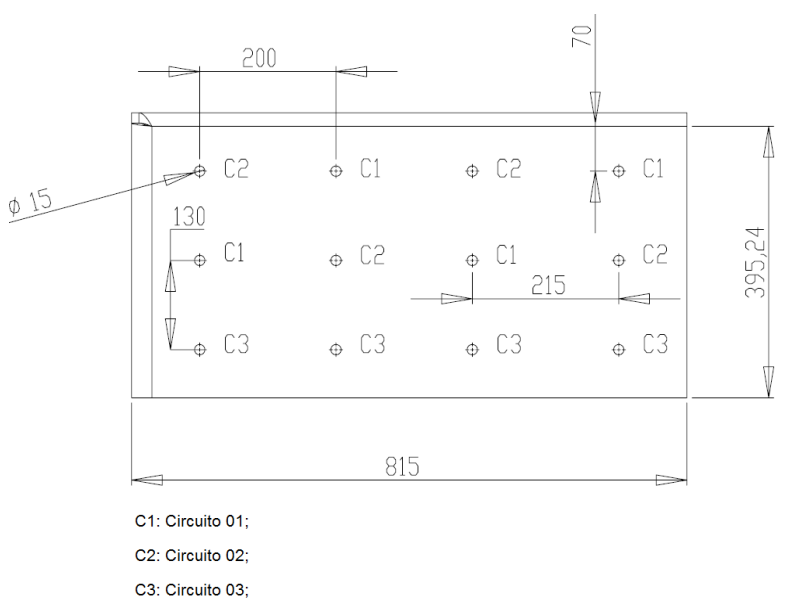

Figura 12 - Distribuição dos circuitos.

\section{ConClusões}

É possivel observar, conforme o que foi apresentado, que a domótica cada vez mais proporciona um maior conforto, comodidade e, principalmente, maior interação com seus usuários. Hoje, indo muito além da segurança e comodidade para os mesmos, permitindo também um melhor aproveitamento de água, gerenciamento a redução do consumo de energia elétrica, recursos estes que cada vez mais caros e escassos em nosso planeta.

Uma das maiores barreiras encontradas ainda para que os sistemas domóticos possam ser mais utilizados tem sido os valores elevados dos equipamentos envolvidos nos sistemas domotizados.

Em suma, este experimento serviu como bom exemplo do estudo de automação residencial, podendo ser otimizado adotando-se outros pontos para tal, resultando em economia e conforto para os usuários.

\section{REFERÊNCIAS}

ANTONELLI, P. L.; Introdução aos Controladores Lógicos Programáveis (CLPS). Disponível em: www.ejm.com.br/download/Introducao\%20CLP.pdf Acesso em: 18 maio. 2011

LUZ, J. M. da.; LUMINOTÉCNICA, 2010. Disponível em:

www.iar.unicamp.br/lab/luz/ld/Livros/Luminotecnica.pdf . Acesso em: 12 maio. 2011.

ABNT - NBR 5413: Iluminância de interiores.Rio de Janeiro, MAIO, 1992. 13 páginas.
NATALE, F.; Automação Industrial. São Paulo, SP, Editora Érica. 2003.

SOUZA ,L.; Edival de. Controladores Lógicos Programáveis. Disponível em: www.leopoldina.cefetmg.br/moodle/file.php/40/clp3.pdf . Acesso em: 06 jun. 2011.

WIKIPEDIA, 2008. image:ReedSwitch.jpg. Disponível em:

http://commons.wikimedia.org/wiki/lmage:ReedSwitch.j pg. Acesso em: 19 maio. 2011. 